\title{
LARGE DEVIATIONS FOR A CATALYTIC FLEMING-VIOT BRANCHING SYSTEM
}

\author{
ILIE GRIGORESCU
}

\begin{abstract}
We consider a jump-diffusion process describing a system of diffusing particles that upon contact with an obstacle (catalyst) die and are replaced by an independent offspring with position chosen according to a weighted average of the remaining particles. The obstacle is a bounded nonnegative function $V(x)$ and the birth/death mechanism is similar to the Fleming-Viot critical branching. Since the mass is conserved, we prove a hydrodynamic limit for the empirical measure, identified as the solution to a generalized semilinear (reaction-diffusion) equation, with nonlinearity given by a quadratic operator. A large deviations principle from the deterministic hydrodynamic limit is provided. The upper bound is given in any dimension, and the lower bound is proven for $d=1$ and $V$ bounded away from zero. An explicit formula for the rate function is provided via an Orlicz type space.
\end{abstract}

\section{INTRODUCTION}

Let $N$ be a positive integer, $\mathbf{T}^{d}$ be the d-dimensional unit torus, $V(x)$ a bounded measurable function on $\mathbf{T}^{d}$, and a scalar function $h \in C^{1,2}\left([0, \infty), \mathbf{T}^{d}\right)$. We shall denote $\mathbf{x}=\left(x_{1}, x_{2}, \ldots, x_{N}\right) \in\left(\mathbf{T}^{d}\right)^{N}$, with the convention that $\mathbf{x}^{i j} \in\left(\mathbf{T}^{d}\right)^{N}$ is the vector where the component $i$ has been deleted and replaced with the component $j$, for all $1 \leq i \neq j \leq N$. In addition, $\{\mathbf{w}(t)\}_{t \geq 0}, \mathbf{w}(t)=\left(w_{1}(t), w_{2}(t), \ldots, w_{N}(t)\right)$ is a system of $N$ independent Brownian motions on $\mathbf{T}^{d}$ with respect to the filtration $\left\{\mathcal{F}_{t}\right\}_{t \geq 0}$ on the probability space $(\Omega, \Sigma, P)$. The probability space accommodates a family of mutually independent Poisson clocks of rate one, also independent of the Brownian motion, enabling the construction of a Feller process $\left\{\mathbf{x}_{V, h}^{N}(t)\right\}_{t \geq 0}$ on the Skorohod space $\mathbf{D}\left([0, \infty),\left(\mathbf{T}^{d}\right)^{N}\right)$, where $\mathbf{x}(t)=\left(x_{1}(t), x_{2}(t), \ldots, x_{N}(t)\right)$ behaves as described in the following. When no confusion on the parameters $(V, h)$ is possible, the process is simply denoted by $\left\{\mathbf{x}^{N}(t)\right\}_{t \geq 0}$. At time $t=0$, the process starts from a distribution on $\left(\mathbf{T}^{d}\right)^{N}$. Write $H(t, \mathbf{x})=\sum_{i=1}^{N} h\left(t, x_{i}\right)$ and

$$
\begin{aligned}
p_{i j}^{N, h}(t, \mathbf{x}(t))=p_{i j} & =(N-1)^{-1} e^{H\left(s, \mathbf{x}^{i j}(s)\right)-H(s, \mathbf{x}(s))} \\
C_{i}^{N}(t, \mathbf{x}(t)) & =\left(\sum_{k \neq i} p_{i j}^{N, h}(t, \mathbf{x}(t))\right) V\left(x_{i}(s)\right) .
\end{aligned}
$$

Each particle $x_{i}(\cdot), 1 \leq i \leq N$, carries a Poisson clock, independent of all others. The particles evolve independently as Brownian motions with drift $\nabla h(s, x)$ until one of the

Date: October 7, 2005.

1991 Mathematics Subject Classification. Primary: 60K35; Secondary: 60J50, 35K15.

Key words and phrases. Fleming-Viot, hydrodynamic limit, large deviations, reaction-diffusion equation, branching. 
clocks with time running at rates $C_{i}^{N}(t, \mathbf{x}(t))$ rings. At that moment, the particle $x_{i}$ jumps to the location of the particle $x_{j}$ with probability $p_{i j}\left(\sum_{k \neq i} p_{i k}\right)^{-1}$. When $h$ is a constant, the particle redistribution is exactly the Fleming-Viot sampling mechanism.

The process $\mathbf{x}(\cdot)$ is the solution of the martingale problem stating that for any $f \in$ $C^{1,2}\left([0, \infty) \times\left(\mathbf{T}^{d}\right)^{N}\right)$, the expression $\mathcal{M}_{f}(t)$ is a $\left(P,\left\{\mathcal{F}_{t}\right\}_{t \geq 0}\right)$ - martingale, where

$$
\begin{aligned}
& \mathcal{M}_{f}(t)=f(t, \mathbf{x}(t))-f(0, \mathbf{x}(0)) \\
& -\int_{0}^{t} \partial_{s} f(s, \mathbf{x}(s))+\sum_{i=1}^{N}\left(\frac{1}{2} \Delta_{x_{i}} f(s, \mathbf{x}(s))+\nabla_{x_{i}} H(s, \mathbf{x}(s)) \cdot \nabla_{x_{i}} f(s, \mathbf{x}(s))\right) d s \\
& -\sum_{i=1}^{N} \int_{0}^{t}\left[\sum_{j \neq i}^{N} p_{i j}^{N, h}(t, \mathbf{x}(t))\left(f\left(s, \mathbf{x}^{i j}(s)\right)-f(s, \mathbf{x}(s))\right)\right] V\left(x_{i}(s)\right) d s .
\end{aligned}
$$

Due to the fact that $\sup _{x \in \mathbf{T}^{d}}|V(x)|<\infty$, the process is well defined for all times, $P-a . s$. . More precisely, it is easy to see that all particles have a finite number of jumps in every finite time interval with probability one, a consequence of the law of large numbers applied to the exponential holding times. In addition, we can write that $\mathcal{M}_{f}^{N}(t)=\mathcal{M}_{f}^{N, B}(t)+\mathcal{M}_{f}^{N, J}(t)$, where $\mathcal{M}_{f}^{N, B}(t)$ is the Brownian martingale and $\mathcal{M}_{f}^{N, J}(t)$ is the jump martingale, with predictable quadratic variations

$$
\begin{aligned}
\left\langle\mathcal{M}_{f}^{N, B}\right\rangle(t) & =\int_{0}^{t} \sum_{i=1}^{N}\left|\nabla_{x_{i}} f(s, \mathbf{x}(s))\right|^{2} d s \\
\left\langle\mathcal{M}_{f}^{N, J}\right\rangle(t) & =\sum_{i=1}^{N} \int_{0}^{t}\left[\sum_{j \neq i} p_{i j}^{N, h}(t, \mathbf{x}(t))\left(f\left(s, \mathbf{x}^{i j}(s)\right)-f(s, \mathbf{x}(s))\right)^{2}\right] V\left(x_{i}(s)\right) d s .
\end{aligned}
$$

The jump-diffusion process $\left\{\mathbf{x}^{N}(\cdot)\right\}$ generalizes the branching mechanism present in the particle-system construction of the catalytic Fleming-Viot measure-valued process. The branching allows conservation of mass, and is triggered by cumulative contact with the catalyst $V$. We choose to keep the particle system perspective on the process. Particles are tagged, and when the exponential clocks ring, they jump to a location of one of the other $N-1$ particles with probabilities weighted according to the corresponding pair involved in the exchange. An interesting point of the large deviations perspective is the natural need to consider inhomogeneous redistribution mechanisms reflected in the probabilities (1.1), and then the jump probabilities from (1.5). By introducing a perturbation of the original system through the factor $\exp \left(H\left(s, \mathbf{x}^{i j}(s)\right)-H(s, \mathbf{x}(s))\right)$, we have to redistribute particle $i$ over to the location of particle $j$ with relative weight $\exp \left(h\left(s, x_{j}(s)\right)\right)$ (after normalization). When $h \equiv 0$, the weights are equal to one, giving the uniform redistribution mechanism of the Fleming-Viot dynamics. An extreme case of the process, introduced in [1], can be described informally by setting $V(x)=+\infty \cdot \mathbf{1}_{D^{c}}$, where $D$ is a bounded region of $\mathbf{R}^{d}$. The large deviations principle in this case should also cover the average number of boundary hits by all particles, as discussed in [5].

The empirical process (1.8) satisfies a law of large numbers (1.15) given in Theorem 1. The deterministic limit is in fact a delta function on the Skorohod space $\mathbf{D}\left([0, \infty), M_{1}\left(\mathbf{T}^{d}\right)\right)$ 
of measure-valued time-indexed paths. Considering the case $h \equiv 0$, we derive large deviations results for the family of empirical processes in Theorem 2. In order to do that, we need a perturbation of the process, by introducing a drift $\nabla h(t, x)$. An exposition of the general method used to derive large deviation principles from hydrodynamic limits is given in [7], for symmetric simple exclusion processes, that appeared in [8]. The closest reference to the present work is [9].

The upper bound is rather standard, leading to a variational formula (1.24) based on (3.13). For the lower bound, there are several important hurdles to overcome. One is to establish the uniqueness of the solution of a nonlinear integro-differential pde (Section 2). The others are specific to the present problem, and cannot be treated with the methods previously available. One has to solve a variational problem in an Orlicz type space (Section 4, Theorem 4, where the bound away from zero of the catalyst $V$ is required) and especially to prove the smoothness of the weak solution of the nonlinear integro-differential equation (5.9). This is done in Section 5, and a certain degree of smoothness is needed for $V$ (continuity), as well as the restriction to $d=1$. The latter is technical, and hopefully can be removed. Finally, we note that Proposition 4 provides the explicit form of the rate function for mildly regular paths $\mu(\cdot, d x)$.

Empirical measure and initial profile. Denote $M_{1}\left(\mathbf{T}^{d}\right)$ the space of probability measures on the $d$-dimensional torus $\mathbf{T}^{d}, \mathbf{D}\left([0, T], \mathbf{T}^{d}\right)$ the Skorohod space of rcll measure-valued paths on the time interval $[0, T]$, where $T>0$ is fixed but arbitrary. The process $\left\{\mathbf{x}^{N}(\cdot)\right\}$ on $\mathbf{D}\left([0, \infty),\left(\mathbf{T}^{d}\right)^{N}\right)$ solving the martingale problem (1.3)-(1.5), induces the empirical measure process

$$
\mu_{h}^{N}(t, d x)=\frac{1}{N} \sum_{i=1}^{N} \delta_{x_{i}(t)} \in \mathbf{D}\left([0, T], \mathbf{T}^{d}\right), \quad t \geq 0 .
$$

Assume that there exists $\mu_{h}(d x) \in M_{1}\left(\mathbf{T}^{d}\right)$ such that the initial empirical distribution converge weakly to $\mu_{h}(d x)$ in probability, in other words, for any $\phi \in C^{1,2}\left([0, T] \times \mathbf{T}^{d}\right)$,

$$
\lim _{\epsilon \rightarrow 0} P\left(\left|\left\langle\phi(0, \cdot), \mu_{h}^{N}(0, \cdot)\right\rangle-\left\langle\phi(0, \cdot), \mu_{h}(\cdot)\right\rangle\right|>\epsilon\right)=0 .
$$

Hydrodynamic limit. Let

$$
\mathcal{L}_{t} \psi=\frac{1}{2} \Delta \psi+\nabla h(t, \cdot) \cdot \nabla \psi, \quad \psi \in C^{1,2}\left([0, T] \times \mathbf{T}^{d}\right)
$$

and

$$
\mathcal{L}_{t}^{*} \psi=\frac{1}{2} \Delta \psi-\nabla \cdot(\psi \nabla h(t, \cdot)), \quad \psi \in C^{1,2}\left([0, T] \times \mathbf{T}^{d}\right)
$$

where $\mathcal{L}_{t}^{*}$ is the adjoint operator of $\mathcal{L}_{t}$. In addition, for any measure $\mu(d x)$, we denote

$$
\mathbf{a}_{h}(\mu ; t, x)=\left\langle e^{-h(t, \cdot)} V(\cdot), \mu(t, \cdot)\right\rangle e^{h(t, x)}-\left\langle e^{h(t, \cdot)}, \mu(t, \cdot)\right\rangle e^{-h(t, x)} V(x)
$$

a linear (also bounded whenever $V$ is bounded) functional on $\mu \in M\left(\mathbf{T}^{d}\right)$ depending on $h(t, x)$, contributing to the nonlinear part of the hydrodynamic limit (1.13). 
Any deterministic measure valued path $\{\mu(t, d x)\}_{t \geq 0}$ verifying the integro-differential equation

$$
\begin{aligned}
& \left\langle\phi(t, \cdot), \mu_{h}(t, \cdot)\right\rangle-\left\langle\phi(0, \cdot), \mu_{h}(0, \cdot)\right\rangle= \\
& \int_{0}^{t}\left\langle\partial_{s} \phi(s, \cdot)+\frac{1}{2} \Delta \phi(s, \cdot)+\nabla \phi(s, \cdot) \cdot \nabla h(s, \cdot), \mu_{h}(s, \cdot)\right\rangle d s+ \\
& \int_{0}^{t} \int_{\mathbf{T}^{d}} \int_{\mathbf{T}^{d}}(\phi(s, y)-\phi(s, x)) e^{h(s, y)-h(s, x)} V(x) \mu_{h}(s, d x) \mu_{h}(s, d y) d s
\end{aligned}
$$

for any $\phi \in C^{1,2}\left([0, T] \times \mathbf{T}^{d}\right)$ is said a weak solution of the nonlinear equation

$$
\partial_{t} \mu=\mathcal{L}^{*} \mu+\mu \mathbf{a}_{h}(\mu) .
$$

Theorem 1. Let the initial configuration $\mu_{h}^{N}(d x)$ of the particles in the process $\mathbf{x}^{N}(\cdot)=$ $\mathbf{x}_{V, h}^{N}(\cdot)$, defined by (1.3)-(1.5) converge weakly in probability to $\mu_{h}(d x) \in M_{1}\left(\mathbf{T}^{d}\right)$ as in (1.9). Assume, in addition, that there exists a bounded function $v_{0}$ on $\mathbf{T}^{d}$ such that $\mu_{h}(0, d x)=$ $v_{0}(x) d x$. Then, for any $\phi \in C^{1,2}\left([0, T] \times \mathbf{T}^{d}\right)$, the empirical measure process $\left\{\mu_{h}^{N}(t, d x)\right\}_{t \geq 0}$ satisfies

$$
\lim _{\epsilon \rightarrow 0} P\left(\sup _{t \in[0, T]}\left|\left\langle\phi(t, \cdot), \mu_{h}^{N}(t, \cdot)\right\rangle-\left\langle\phi(t, \cdot), \mu_{h}(t, \cdot)\right\rangle\right|>\epsilon\right)=0,
$$

where the limiting measure valued process $\left\{\mu_{h}(t, \cdot)\right\}_{t \in[0, T]}$ is concentrated on the deterministic path given by the unique weak solution of the partial differential equation (1.13) with initial profile $\mu_{h}(0, \cdot)=\mu_{h}(\cdot)$.

Proof. For a smooth $\phi \in C^{1,2}\left([0, T], \mathbf{T}^{d}\right)$, we apply Ito's formula for semi-martingales to the test function $\left\langle\phi(t, x), \mu_{h}^{N}(t, d x)\right\rangle=N^{-1} \sum_{j} \phi\left(t, x_{j}(t)\right)$, with $\mu_{h}^{N}$ given in (1.8). Due to the mean field character of the evolution, the calculation is straightforward (see also [6], and [5] for the related limit for hard obstacle $V$ ). The martingale converges to zero, in square norm. Moreover, the quadratic variation over time intervals $[s, t], 0 \leq s<t \leq T$ is bounded uniformly in $N$ by a modulus of continuity that vanishes as $t-s \rightarrow 0$, proving that the empirical measure process is tight in the Skorohod space, with time-continuous limit $\mu_{h}(\cdot, d x) \in C\left([0, T], M_{1}\left(\mathbf{T}^{d}\right)\right)$. We derive that any limit point $\mu_{h}(t, d x)$ of the tight sequence of empirical measure processes indexed by $t$ satisfies the weak pde (1.13). The proof is complete if (1.13) has a unique solution, which is shown in Theorem 3.

Large deviations principle. Given $\mu(d x), \mu_{0}(d x)$ in $M_{1}\left(\mathbf{T}^{d}\right), H\left(\mu \mid \mu_{0}\right)$ is the relative entropy of $\mu$ given $\mu_{0}$, or

$$
H\left(\mu \mid \mu_{0}\right)=\sup _{q \in C^{2}\left(\mathbf{T}^{d}\right)} \int_{\mathbf{T}^{d}} q(x) \mu(d x)-\log \left(\int_{\mathbf{T}^{d}} e^{q(x)} \mu_{0}(d x)\right)
$$

equal to

$$
H\left(\mu \mid \mu_{0}\right)=\left\{\begin{array}{rl}
\int_{\mathbf{T}^{d}} \frac{d \mu(d x)}{d \mu_{0}(d x)} d x, & \text { if } \mu(d x)<<\mu_{0}(d x), \\
+\infty & \text { otherwise }
\end{array} .\right.
$$


For any $\mu \in C\left([0, T], \mathbf{M}_{1}\left(\mathbf{T}^{d}\right)\right), \psi \in C\left([0, T], \mathbf{T}^{d}\right), t \in[0, T]$, we have $\langle\psi(t), \mu(t)\rangle=$ $\int_{\mathbf{T}^{d}} \psi(t, x) \mu(t, d x) d x$. Let $\phi \in C^{1,2}\left([0, T], \mathbf{T}^{d}\right)$, and write

$$
\begin{aligned}
\ell_{\phi}(\mu) & =\langle\phi(T), \mu(T)\rangle-\langle\phi(0), \mu(0)\rangle \\
& -\int_{0}^{T}\left\langle\partial_{s} \phi(s), \mu(s)\right\rangle+\left\langle\frac{1}{2} \Delta \phi(s), \mu(s)\right\rangle d s
\end{aligned}
$$

and

$$
\begin{aligned}
J_{\phi}(\mu) & =\ell_{\phi}(\mu) \\
& -\frac{1}{2} \int_{0}^{T}\left\langle|\nabla \phi(s)|^{2}, \mu(s)\right\rangle d s \\
& -\int_{0}^{T} \int_{\mathbf{T}^{d}} \int_{\mathbf{T}^{d}}\left(e^{\phi(s, y)-\phi(s, x)}-1\right) V(x) \mu(s, d y) \mu(s, d x) d s .
\end{aligned}
$$

We are ready to write down the rate function:

$$
I(\mu)=I_{\text {init }}(\mu)+I_{d y n}(\mu) \quad \text { if } \quad \mu \in \mathbf{D}\left([0, T], \mathbf{M}_{1}\left(\mathbf{T}^{d}\right),\right.
$$

where

$$
I_{\text {init }}(\mu)=H\left(\mu \mid \mu_{0}\right), \quad I_{d y n}(\mu)=\sup _{\phi \in C^{1,2}\left([0, T] \times \mathbf{T}^{d}\right)} J_{\phi}(\mu) .
$$

We note that $I(\mu)$ may take the value $+\infty$ and point to Proposition 4 , which provides the explicit form of the rate function for paths $\mu(\cdot, d x)=\mu_{h}(\cdot, d x)$ that arise as hydrodynamic limits of a perturbed process as in Theorem 1.

Theorem 2. The empirical measures $\left\{\mu^{N}(\cdot, d x)\right\}_{N>0}$ defined in (1.8) of the process solving the martingale problem (1.3)-(1.5) for $h \equiv 0$ satisfy a law of large numbers (1.15) as in Theorem 1. In addition, in any dimension d, given that $V$ is bounded, then for any closed subset $\mathcal{G}$ of $\mathbf{D}\left([0, T], M^{1}\left(\mathbf{T}^{d}\right)\right)$

$$
\limsup _{N \rightarrow \infty} \frac{1}{N} \log Q^{N}\left(\mu^{N} \in \mathcal{G}\right) \leq-\inf _{\mu \in \mathcal{G}} I(\mu)
$$

and, if $d=1$, given that $V \in C\left(\mathbf{T}^{d}\right)$ and there exists $c_{-}>0$ such that $V(x) \geq c_{-}$for all $x \in \mathbf{T}^{d}$, then for any open subset $\mathcal{O}$ of $\mathbf{D}\left([0, T], M^{1}\left(\mathbf{T}^{d}\right)\right)$,

$$
\liminf _{N \rightarrow \infty} \frac{1}{N} \log Q^{N}\left(\mu^{N} \in \mathcal{O}\right) \geq-\inf _{\mu \in \mathcal{O}} I(\mu) .
$$

Remark. 1) In dimension $d=1$, when $V$ is continuous and bounded away from zero, a complete large deviations principle is satisfied with rate function $I(\cdot)$, given by $(1.23) .2$ ) We can also check that in Proposition $4, I_{d y n}\left(\mu_{h}\right)=0$ when $h \equiv 0$. 3) The proof remains valid when the obstacle $V$ depends on time as well. In this case we require boundedness for the upper bound and $C^{2}$ smoothness in $t$, in addition to the requirements of the theorem, for the lower bound.

Proof. Section 3 proves the upper bound (1.25) and Section 5 proves the lower bound (1.26). 


\section{The PDE.}

Theorem 3. Any weak solution $\mu_{h}(t, d x)$ in the sense of (1.13) of the equation (1.14) with initial value $\mu_{h}(0, d x)=\mu_{h}(d x) \in M\left(\mathbf{T}^{d}\right)$ is a bounded function $\mu_{h}(t, d x)=v_{h}(t, x) d x$ for $t>0$ with a uniform bound $c(t)$ depending only on the time $t$. If the initial value is a bounded function $\mu_{h}(d x)=v_{0, h}(x) d x$ and $0 \leq v_{0, h} \leq c$ then the solution is unique.

Proof. We shall prove first that the solution is a function, and then that it is unique.

The solution is a bounded function. Let $p_{h}(t, x, y)$ be the kernel of the semigroup with generator $\mathcal{L}$ from (1.10), $C$ such that $\mathbf{a}_{h}(\mu ; t, x) \leq C$ uniformly in $\mu, t, x$ and $\tilde{p}_{h}(t, x, y)=$ $e^{C t} p_{h}(t, x, y)$. For $r>0, t>0$ such that $t+r \leq T$ and any $g \in L^{1}\left(\mathbf{T}^{d}\right)$ define the function in $C^{1,2}\left([0, t] \times \mathbf{T}^{d}\right)$

$$
\tilde{g}(s, x)=\int_{\mathbf{T}^{d}} g(y) \tilde{p}_{h}(t+r-s, x, y) d y .
$$

We set $\tilde{g}=\phi$ in (1.13) and obtain

$$
\begin{aligned}
& \left\langle\tilde{g}(t, \cdot), \mu_{h}(t, \cdot)\right\rangle=\left\langle\tilde{g}(0, \cdot), \mu_{h}(0, \cdot)\right\rangle \\
& +\int_{0}^{t} \int_{\mathbf{T}^{d}}\left(\mathbf{a}_{h}(\mu ; s, x)-C\right) \tilde{g}(s, x) \mu_{h}(s, d x) d s
\end{aligned}
$$

which proves

$$
\left\langle\tilde{g}(t, \cdot), \mu_{h}(t, \cdot)\right\rangle \leq\left\langle\tilde{g}(0, \cdot), \mu_{h}(0, \cdot)\right\rangle \leq c(t+r) \int_{\mathbf{T}^{d}}|g(y)| d y
$$

for any $g \geq 0$, where $c(t)=e^{C t} \sup _{x, y \in \mathbf{T}^{d}} p_{h}(t, x, y), t>0$. Let $g(x)=\mathbf{1}_{G}(x)$ where $G$ is an open set in $\mathbf{T}^{d}$. Fatou's lemma applied to $\left|\int_{\mathbf{T}^{d}} \tilde{g}(t, x) \mu_{h}(t, d x)\right|$ as $r \rightarrow 0$ shows that, for $g(y)=\mathbf{1}_{G}(y)$ and $t>0$,

$$
\mu_{h}(t, G) \leq \liminf _{r \rightarrow 0} \int_{\mathbf{T}^{d}} \tilde{g}(t, x) \mu_{h}(t, d x) \leq c(t) \int_{\mathbf{T}^{d}}|g(y)| d y=c(t)|G| .
$$

In case $\mu_{h}(d x)=v_{0, h}(x) d x$ is a bounded function, we re-calculate the right hand side of $(2.2)$

$$
\left\langle\tilde{g}(0, \cdot), \mu_{h}(0, \cdot)\right\rangle=\int_{\mathbf{T}^{d}} \int_{\mathbf{T}^{d}} g(y) \tilde{p}_{h}(t+r, x, y) v_{0, h}(x) d y d x \leq c \int_{\mathbf{T}^{d}}|g(y)| d y=c|G|
$$

which concludes the first part of the proof.

Uniqueness. We prove uniqueness under the assumption of uniform boundedness of the solutions. Let $c$ be such that the two solutions $\mu_{h}^{1}(t, d x)=v_{h}^{1}(x) d x$ and $\mu_{h}^{2}(t, d x)=v_{h}^{2}(x) d x$ satisfy $0 \leq v_{h}^{1}(x) \leq c$ and $0 \leq v_{h}^{2}(x) \leq c$. Equation (1.14) is a reaction-diffusion integrodifferential equation. We show that the nonlinear term $v \mathbf{a}_{h}(v)$ is uniformly bounded and Lipschitz. For any $t \geq 0, i=1,2$, in the uniform norm $\|\cdot\|$

$$
\left\|v^{i} \mathbf{a}_{h}\left(v^{i}\right)\right\| \leq 2 c^{2} \exp (2\|h\|)\|V\|
$$


from (1.12). In addition,

$$
\begin{aligned}
\left\|v^{1} \mathbf{a}_{h}\left(v^{1}\right)-v^{2} \mathbf{a}_{h}\left(v^{2}\right)\right\| \leq & \\
& \left\|v^{1}-v^{2}\right\| \sup _{x \in \mathbf{T}^{d}}\left|\mathbf{a}_{h}\left(v^{1} ; t, x\right)\right| \\
& +\left\|v^{2}\right\| \sup _{x \in \mathbf{T}^{d}}\left|\mathbf{a}_{h}\left(v^{1} ; t, x\right)-\mathbf{a}_{h}\left(v^{2} ; t, x\right)\right| .
\end{aligned}
$$

According to (1.12), line (2.7) is bounded above by $2 c \exp (2\|h\|)\|V\|\left\|v^{1}-v^{2}\right\|$ while $\left\|v^{2}\right\| \leq c$ and

$$
\sup _{x \in \mathbf{T}^{d}}\left|\mathbf{a}_{h}\left(v^{1} ; t, x\right)-\mathbf{a}_{h}\left(v^{2} ; t, x\right)\right| \leq 2 e^{2\|h\|}\|V\|\left\|v^{1}-v^{2}\right\|
$$

provide a Lipschitz bound for line (2.8). The rest of the proof follows identically from Oelschläger [11], Proposition 3.4 and 3.5.

\section{The Upper Bound.}

We apply the integral formula (1.3)-(1.5) in the case $h(t, x) \equiv 0$ to the function $f(t, \mathbf{x})=$ $\exp \left(\sum_{i=1}^{N} \phi\left(t, x_{i}\right)\right)$, where $\phi \in C^{1,2}\left([0, T] \times \mathbf{T}^{d}\right)$. With the observation

$$
f(t, \mathbf{x}(t))=N\left\langle\phi(t, \cdot), \mu^{N}(t, \cdot)\right\rangle=\left\langle\phi(t), \mu^{N}(t)\right\rangle
$$

for simplification, we see that

$$
\exp N\left\langle\phi(t), \mu^{N}(t)\right\rangle-\exp N\left\langle\phi(0), \mu^{N}(0)\right\rangle
$$

$$
-\int_{0}^{t} e^{N\left\langle\phi(s), \mu^{N}(s)\right\rangle}\left\{N\left[\left\langle\partial_{s} \phi(s), \mu^{N}(s)\right\rangle+\left\langle\frac{1}{2} \Delta \phi(s), \mu^{N}(s)\right\rangle+\left\langle|\nabla \phi(s)|^{2}, \mu^{N}(s)\right\rangle\right]\right\} d s
$$

$$
-\int_{0}^{t} \frac{N^{2}}{N-1} e^{N\left\langle\phi(s), \mu^{N}(s)\right\rangle}\left\{\left\langle e^{\phi(s)}, \mu^{N}(s)\right\rangle\left\langle V e^{-\phi(s)}, \mu^{N}(s)\right\rangle-\left\langle V, \mu^{N}(s)\right\rangle\right\} d s
$$

is a martingale. We notice that we can write the last integrand as

$$
\int_{\mathbf{T}^{d}} \int_{\mathbf{T}^{d}}\left(e^{\phi(s, y)-\phi(s, x)}-1\right) V(x) \mu^{N}(s, d y) \mu^{N}(s, d x) .
$$

For any $\mu \in C\left([0, T], \mathbf{M}_{1}\left(\mathbf{T}^{d}\right)\right.$, recall (1.18) and (1.20). If

$$
\mathcal{E}_{\phi}(\mu)=-\int_{0}^{t} \frac{1}{N-1}\left\{\left\langle e^{\phi(s)}, \mu(s)\right\rangle\left\langle V e^{-\phi(s)}, \mu(s)\right\rangle-\langle V, \mu(s)\rangle\right\} d s
$$

then, the formula of the martingale (3.1) and the differential formula for semimartingales show that

$$
\exp \left\{N\left(J_{\phi}\left(\mu^{N}\right)+\mathcal{E}_{\phi}\left(\mu^{N}\right)\right)\right\}
$$


is an exponential martingale with mean one. At time $t=T$,

$$
E^{P^{N}}\left[\exp \left\{N\left(J_{\phi}\left(\mu^{N}\right)+\mathcal{E}_{\phi}\left(\mu^{N}\right)\right)\right\} \exp \left\{N\left\langle q(\cdot), \mu^{N}(0)\right\rangle\right\}\right]=\exp \left\{N \log \int_{\mathbf{T}^{d}} e^{q(x)} d x\right\}
$$

proving that, for

$$
J_{q}^{0}(\mu)=\langle q, \mu(0)\rangle-\log \int_{\mathbf{T}^{d}} e^{q(x)} d x
$$

and

$$
\begin{gathered}
\tilde{J}_{q, \phi}(\mu)=J_{\phi}(\mu)+J_{q}^{0}(\mu), \\
\exp \left\{N\left(\tilde{J}_{q, \phi}\left(\mu^{N}\right)+\mathcal{E}_{\phi}\left(\mu^{N}\right)\right)\right\}
\end{gathered}
$$

is a mean one exponential martingale. From (3.5), we see that, for sufficiently large $N$,

$$
\left\|\mathcal{E}_{\phi}(\mu)\right\| \leq N^{-1} c(\phi), \quad c(\phi)=\|V\|\left(e^{2\|\phi\|}+1\right) .
$$

Let $\mathcal{A}$ be a Borel set in $\mathbf{D}\left([0, T], \mathbf{M}_{1}\left(\mathbf{T}^{d}\right)\right)$. Then

$$
\begin{aligned}
& Q^{N}\left(\mu^{N} \in \mathcal{A}\right)= \\
& E^{Q^{N}}\left[\exp \left\{-N\left(\tilde{J}_{q, \phi}\left(\mu^{N}\right)+\mathcal{E}_{\phi}\left(\mu^{N}\right)\right)\right\} \cdot \exp \left\{N\left(\tilde{J}_{q, \phi}\left(\mu^{N}\right)+\mathcal{E}_{\phi}\left(\mu^{N}\right)\right)\right\} \mathbf{1}_{\left\{\mu^{N} \in \mathcal{A}\right\}}\right] \\
& \leq E^{Q^{N}}\left[\exp \left\{-N\left(\tilde{J}_{q, \phi}\left(\mu^{N}\right)-\frac{c(\phi)}{N}\right)\right\} \cdot \exp \left\{N\left(\tilde{J}_{q, \phi}\left(\mu^{N}\right)+\mathcal{E}_{\phi}\left(\mu^{N}\right)\right) \mathbf{1}_{\left\{\mu^{N} \in \mathcal{A}\right\}}\right\}\right] \\
& \left.\leq \exp \left\{-N \inf _{\mu \in \mathcal{A}}\left\{\tilde{J}_{q, \phi}(\mu)\right\}+c(\phi)\right)\right\}
\end{aligned}
$$

which implies

$$
\limsup _{N \rightarrow \infty} \frac{1}{N} \log Q^{N}\left(\mu^{N} \in \mathcal{A}\right) \leq-\sup _{q, \phi} \inf _{\mu \in \mathcal{A}}\left\{\tilde{J}_{q, \phi}(\mu)\right\}
$$

The three terms defining $J_{\phi}(\mu)$ in (1.20) are continuous as functionals on $\mathbf{D}\left([0, T], \mathbf{M}_{1}\left(\mathbf{T}^{d}\right)\right)$. The first (1.20) is linear. The second (1.21) and third (1.22) terms are continuous as follows. Suppose $\lim _{l \rightarrow \infty} \mu_{l}=\mu$ weakly in the Skorohod norm. This implies that for any continuous and bounded function, as $\nabla \phi$ and the integrand (3.4) present in (3.5), the rcll paths $s \rightarrow\left\langle|\nabla \phi(s)|^{2}, \mu_{l}(s)\right\rangle$ and (3.4) indexed by $l$ converge to the corresponding paths for the limit $\mu$. The functionals are uniformly bounded for each fixed test function $\phi$ and convergence in the Skorohod topology implies almost sure convergence in time. The continuity follows by dominated convergence. In addition, (3.8) is continuous as well. The set $\mathcal{A}$ can be taken to be a compact $\mathcal{K}$. At this point we can use standard results (see Kipnis-Landim [6], Appendix 2, Lemma 3.3) to conclude that

$$
\limsup _{N \rightarrow \infty} \frac{1}{N} \log Q^{N}\left(\mu^{N} \in \mathcal{K}\right) \leq-\inf _{\mu \in \mathcal{K}} I(\mu)
$$

where $I(\mu)=I_{\text {init }}(\mu)+I_{d y n}(\mu)$ given in (1.23), for the case when $\mu_{0}(d x)=v_{0} d x$. 


\section{Orlicz norms.}

Let $t>0$ fixed but arbitrary, $\mu \in C\left([0, t], M_{1}\left(\mathbf{T}^{d}\right)\right)$ and consider the space $H_{t}^{1}(\mu)$ of real-valued measurable functions $f$ on $[0, t] \times \mathbf{T}^{d}$ such that

$$
\int_{0}^{t} \int_{\mathbf{T}^{d}}\left[|f(s, x)|^{2}+|\nabla f(s, x)|^{2}\right] \mu(s, d x) d s<\infty .
$$

Given a real-valued measurable function $\rho$, we define the functional

$$
\begin{array}{r}
\Phi_{\rho, V}(f)=\int_{0}^{t}\left\{\frac{1}{2} \int|\nabla f(s, x)|^{2} \mu(d x)\right. \\
\left.+\iint \rho(f(s, y)-f(s, x)) V(x) \mu(s, d y) \mu(s, d x)\right\} d s
\end{array}
$$

on $f \in H_{t}^{1}(\mu)$, whenever it makes sense.

We shall consider the case of $\rho(x)$ equal to one of the functions $\gamma(x)=\cosh x-1$, its convex conjugate $\gamma^{*}(x)$, and $\zeta(x)=e^{x}-1-x$. Notice that $\gamma, \gamma^{*}, \zeta$ are (i) convex with effective domain $\mathbb{R}$, (ii) equal to zero at zero, (iii) positive everywhere else. In addition, $\zeta$ is increasing everywhere, while $\gamma, \gamma^{*}$ are even, increasing for $x>0$ and represent a pair of conjugate $N$-functions. We note that $\gamma \in \Delta_{2}, \gamma^{*} \in \nabla_{2}$ (for this and the definitions of Young functions the reader is refereed to [12]).

Let $\Phi$ be one of the functionals defined above. On the space

$$
\tilde{U}_{\Phi}=\{f \text { measurable : } \exists \lambda>0 \quad \Phi(\lambda f)<\infty\}
$$

we define the functionals

$$
\|f\|_{\Phi}:=\inf \left\{\beta>0: \Phi\left(\frac{f}{\beta}\right) \leq 1\right\} .
$$

Since $\mu(s, d x)$ is a finite measure, all bounded functions are in $\tilde{U}_{\Phi}$.

Proposition 1. When $\Phi$ is determined by a Young function $\rho(x)$, the functionals (4.2) are semi-norms on $\tilde{U}_{\Phi}$. Let $U_{\Phi}$ denote the quotient space obtained by identifying functions $f \in \tilde{U}_{\Phi}$ that differ by a constant $\mu(d s, d x)$ - almost surely. Then $U_{\Phi}$ is a Banach space with norm $\|f\|_{\Phi}$.

Proof. The proof is straightforward and left to the reader.

Proposition 2. If $V(x)$ is bounded above and below, then there exists constants $0<c_{1} \leq c_{2}$ such that

$$
\Phi_{\gamma, c_{1}}(f) \leq \Phi_{\rho, V}(f) \leq \Phi_{\gamma, c_{2}}(f) .
$$

For any $\left.f \in C^{\infty}\left([0, t], \mathbf{T}^{d}\right)\right)$, we shall write

$$
\begin{aligned}
\ell(f) & =\int_{D} f(t, x) \mu(t, d x)-\int_{D} f(0, x) \mu(0, d x) \\
& -\int_{0}^{t} \int_{D} \partial_{s} f(s, x)+\frac{1}{2} \Delta f(s, x) \mu(s, d x) d s \\
& -\int_{0}^{t} \int_{D} \int_{D}(f(s, y)-f(s, x)) V(x) \mu(s, d x) \mu(s, d y) .
\end{aligned}
$$


We want to show the following result.

Theorem 4. For any fixed $t>0$, any finite measure $\mu(\cdot, \cdot)$ on $[0, t] \times \mathbf{T}^{d}$, the variational problem

$$
I_{d y n}(\mu)=\sup _{\left.f \in C^{\infty}\left([0, t], \mathbf{T}^{d}\right)\right)}\left\{\ell(f)-\Phi_{\zeta, V}(f)\right\}<\infty
$$

implies that $\ell \in U_{\Phi_{\gamma, 1}}^{*}$ and the supremum is achieved for a function $h=h_{\mu} \in U_{\Phi_{\gamma, 1}}$ for which $\mu$ is a weak solution of the semi-linear equation (1.14). The star superscript denotes the topological dual of $U_{\Phi_{\gamma, 1}}$.

Proof. The proof is given after Lemma 1 and Proposition 3.

Lemma 1. The space $U_{\Phi_{\rho, 1}}$ is a dual space. More concretely, $U_{\Phi_{\rho^{*}, 1}^{*}}=U_{\Phi_{\rho, 1}}$.

Proof. Young's inequality $x y \leq \gamma(x)+\gamma^{*}(y)$ for a Young function $\gamma$ and its convex conjugate $\gamma^{*}$ implies that the bilinear functional

$$
\begin{array}{r}
Q(f, g)=\int_{0}^{t} \int \nabla f(s, x) \nabla g(s, x) \mu(s, d x) d s \\
+\frac{1}{2} \int_{0}^{t} \iint(f(s, y)-f(s, x))(g(s, y)-g(s, x)) \mu(s, d x) \mu(s, d y) d s
\end{array}
$$

is finite for any $f \in U_{\Phi_{\rho^{*}, 1}}$ and $g \in U_{\Phi_{\rho, 1}}$. A first classical fact we need is that the new functional

$$
\|f\|_{\Phi_{\rho, 1}}^{\prime}=\sup \left\{|Q(f, g)|: \Phi^{*}(g) \leq 1\right\}
$$

is an equivalent norm to $\|f\|_{\Phi_{\rho, 1}}$ on $\tilde{U}_{\Phi_{\rho, 1}} / \mathcal{C}$, where $\mathcal{C}$ is the class of functions that differ by a constant $\mu$-almost surely. We refer the reader to Proposition 3.3.4, page 61 from [12].

The next step is based on the observation that $\gamma^{*}(x)=x \ln \left(x+\sqrt{x^{2}+1}\right)-\sqrt{x^{2}+1}+1 \leq$ $p(x)=\frac{1}{2} x^{2}$, which implies that $U_{\Phi_{p, 1}} \subseteq U_{\Phi_{\rho^{*}, 1}}$ and follows an idea from P. Lax [10], p. 79. A bounded linear functional $\alpha \in U_{\Phi_{\rho^{*}, 1}}^{*}$ satisfies $|\alpha(f)| \leq K\|f\|_{\Phi_{\rho^{*}, 1}}$ for some $K>0$ and any $f \in U_{\Phi_{\rho^{*}, 1}}$. The previous observation and the fact that $\|f\|_{\Phi_{\rho^{*}, 1}} \leq\|f\|_{\Phi_{p, 1}}$ show that $\alpha$ is a bounded linear functional on $U_{\Phi_{\rho, 1}}$. The space generated by the function $p(x)$ is a Hilbert space, since $Q(\cdot, \cdot)$ is an inner product, and we identify functions $f_{1}, f_{2}$ with $f_{1}-f_{2} \in \mathcal{C}$. There exists a function $g \in U_{\Phi_{p, 1}}$ such that, for any $f \in U_{\Phi_{p, 1}} \subseteq U_{\Phi_{\rho^{*}, 1}}$, $\alpha(f)=Q(f, g)$. Note that $\left.C^{\infty}\left([0, t], \mathbf{T}^{d}\right)\right)$ is dense in $U_{\Phi_{\rho^{*}, 1}}$. This allows to take the supremum in the norm (4.7) over $\left.C^{\infty}\left([0, t], \mathbf{T}^{d}\right)\right) \subseteq U_{\Phi_{p, 1}}$ (to ensure the validity of the inclusions, we remind that the set $\mathbf{T}^{d}$ ) is a bounded region in $\mathbb{R}^{d}$ ). At this point, since the norm (4.7) is equivalent to $\|\cdot\|_{\Phi_{\rho, 1}}$, there exists a constant $K_{1}>0$ such that

$$
\left.K_{1}\|g\|_{\Phi_{\rho, 1}} \leq \sup \left\{|Q(f, g)|: f \in C^{\infty}\left([0, t], \mathbf{T}^{d}\right)\right),\|f\|_{\Phi_{\rho^{*}, 1}} \leq 1\right\} .
$$

Since $\left.C^{\infty}\left([0, t], \mathbf{T}^{d}\right)\right) \subseteq U_{\Phi_{p, 1}}$, the equality $\alpha(f)=Q(f, g)$ holds. We know that $|\alpha(f)| \leq$ $K\|f\|_{\Phi_{\rho^{*}, 1}}$. This implies that $\|g\|_{\Phi_{\rho, 1}} \leq K / K_{1}<\infty$, which is what we wanted to prove. 
Proposition 3. 1) The functional $\Phi_{\zeta, V}(\cdot)$ is lower semi-continuous on $U_{\Phi_{\gamma, 1}}$.

2) If $\ell(\cdot)$ is a continuous linear functional on $U_{\Phi_{\gamma, 1}}$, then for any real $q$, the sets

$$
K_{q}=\left\{f \in U_{\Phi_{\rho, 1}}: \ell(f)-\Phi_{\zeta, V}(f) \geq q\right\}
$$

are weak* - compact in $U_{\Phi_{\rho, 1}}$ in the topology induced by $\sigma\left(U_{\Phi_{\rho, 1}}, U_{\Phi_{\rho^{*}, 1}}\right)$.

Proof. Since the closed ball centered at zero is compact in the weak* topology, one has to show that 1$)$ the mapping $f \longrightarrow \ell(f)-\Phi_{\zeta, V}(f)$ is lower semi-continuous and 2) that there exists a constant $C_{1}>0$ such that $f \in K_{q}$ implies that $\|f\|_{\Phi_{\rho, c_{2}}} \leq C_{1}$.

For 1) we remind that $\gamma$ is convex with effective domain $\operatorname{dom}(\gamma)=\mathbb{R}$. The norms $\|\cdot\|_{\Phi_{\rho, c}}$ are equivalent for all $c>0$ so we shall use the norm corresponding to $c=1$. Assume $\left\|f_{n}-f\right\|_{\Phi_{\rho, 1}} \rightarrow 0$ as $n \rightarrow \infty$ in $U_{\Phi_{\gamma, 1}}$. Since $\gamma(x)=\cosh x-1 \geq p(x)=\frac{1}{2} x^{2}$, it follows that $U_{\Phi_{\gamma, 1}} \subseteq U_{\Phi_{p, 1}}$ and $\left\|f_{n}-f\right\|_{\Phi_{p, 1}} \rightarrow 0$. The gauge norm associated to $p(x)$ generates a Hilbert space, which implies that there exists a subsequence $f_{n^{\prime}}$ over which $\nabla f_{n^{\prime}} \rightarrow \nabla f$ and also that

$$
\lim _{n^{\prime} \rightarrow \infty} \int_{0}^{t} \iint\left(\check{f}_{n^{\prime}}(s, x, y)-\check{f}(s, x, y)\right)^{2} V(x) \mu(s, d x) \mu(s, d y) d s=0,
$$

where $\check{g}(s, x, y)=g(s, y)-g(s, x)$ for any $g \in U_{\Phi_{p, 1}}$. This implies that there is a subsequence of $f_{n^{\prime}}$ indexed by $n^{\prime \prime}$ over which $\check{f}_{n^{\prime \prime}} \rightarrow \check{f}$ almost surely with respect to the measure $V(x) \mu(s, d x) \otimes \mu(s, d y) \otimes d s$. To simplify notation, we denote $f_{n^{\prime \prime}}$ by $f_{n}$ in the following. Furthermore, there exists at least one $y_{0} \in \mathbf{T}^{d}$ ) (actually a set of measure one of them) such that $\check{f}_{n}\left(s, x, y_{0}\right) \rightarrow \check{f}\left(s, x, y_{0}\right)$ on a set of measure one with respect to $V(x) \mu(s, d x) \otimes d s$, so $f_{n}(s, x) \rightarrow f(s, x)$ a.s. relative to $\mu(s, d x) \otimes d s$, since $V$ is bounded below. The functions $\zeta\left(f_{n}(s, y)-f_{n}(s, x)\right)$ and $\left|\nabla f_{n}(s, x)\right|^{2}$ are nonnegative and continuous. By the Fatou lemma we have

$$
\liminf _{n \rightarrow \infty} \Phi_{\zeta, V}\left(f_{n}\right) \geq \Phi_{\zeta, V}(f),
$$

which is what we needed to prove.

For 2) we write that $f \in K_{q}$ implies

$$
\Phi_{\rho, c_{1}}(|f|)=\Phi_{\rho, c_{1}}(f) \leq \Phi_{\xi, V}(f) \leq \ell(f)-q \leq C\|f\|_{\Phi_{\rho, c_{2}}}+|q| \leq C^{\prime}\|f\|_{\Phi_{\rho, c_{1}}}+|q|,
$$

where $C^{\prime}$ was introduced to compensate for the switch from $c_{2}$ to $c_{1}$ in the equivalent norms $\|f\|_{\Phi_{\rho, c_{i}}}, i=1,2$, and the absolute value on the left hand side of the inequality is permitted due to the evenness of $\gamma$. Assume there exists no constant $C_{1}$ as above. Then there exists a sequence $\left\{f_{n}\right\} \in K_{q}$ with $\left\|f_{n}\right\|_{\Phi_{\rho, c_{1}}} \rightarrow \infty$. Write $\lambda_{n}=\left\|f_{n}\right\|_{\Phi_{\rho, c_{1}}}-1$. For large $n, \lambda_{n}>>1$. The elementary inequality

$$
\rho(\lambda x)=\cosh (\lambda x)-1 \geq \lambda^{2} \rho(x), \quad x \geq 0, \quad \lambda \geq 1
$$

applied to the integral (4.1) defining the functional $\Phi_{\rho, c_{1}}$ with $\lambda \rightarrow \lambda_{n}$ and $x \rightarrow|f| / \lambda_{n}$ gives

$$
\lambda_{n}^{2} \Phi_{\rho, c_{1}}\left(\frac{\left|f_{n}\right|}{\lambda_{n}}\right) \leq C^{\prime}|| f \|_{\Phi_{\rho, c_{1}}}+|q| .
$$

The definition of the gauge norm (4.2) and the fact that $0<\lambda_{n}<\left\|f_{n}\right\|_{\Phi_{\rho, c_{1}}}$ imply that either $\Phi_{\rho, c_{1}}\left(\frac{\left|f_{n}\right|}{\lambda_{n}}\right)=+\infty$, in which case we obtained a contradiction, or $\Phi_{\rho, c_{1}}\left(\frac{\left|f_{n}\right|}{\lambda_{n}}\right)>1$. In 
the latter case, we obtained that $\left(\left\|f_{n}\right\|_{\Phi_{\rho, c_{1}}}-1\right)^{2} \leq C^{\prime}\left\|f_{n}\right\|_{\Phi_{\rho, c_{1}}}+|q|$, which contradicts the fact that the norms of $f_{n}$ tend to infinity.

4.1. Proof of Theorem 4. Part 1. We first show that $\ell \in U_{\Phi_{\gamma, 1}}^{*}$. Due to the right-hand side of inequality (4.3), the supremum in (4.5) remains finite when we substitute $\Phi_{\zeta, V}$ by $\Phi_{\gamma, c_{2}}$. If

$$
\sup _{\left.f \in C^{\infty}\left([0, t], \mathbf{T}^{d}\right)\right)}\left\{\ell(f)-\Phi_{\gamma, c_{2}}(f)\right\}=C_{0}<\infty
$$

then there exists $C>0$ such that $|\ell(f)| \leq C\|f\|_{\Phi_{\gamma, c_{2}}}$, in other words $\ell$ is continuous in the Orlicz norm $\|\cdot\|_{\Phi_{\gamma, c_{2}}}$. We prove this by contradiction. Assume there exists a sequence $\left\{f_{n}\right\}$ such that $\left|\ell\left(f_{n}\right)\right|>n\left\|f_{n}\right\|_{\Phi_{\gamma, c_{2}}}$, or $\left\|f_{n}\right\|_{\Phi_{\gamma, c_{2}}}<n^{-1}\left|\ell\left(f_{n}\right)\right|$. By the definition of the Orlicz norm,

and we can write

$$
\Phi_{\gamma, c_{2}}\left(\frac{n f_{n}}{\left|\ell\left(f_{n}\right)\right|}\right) \leq 1
$$

and then

$$
\left(\frac{n}{\left|\ell\left(f_{n}\right)\right|}\right) \ell\left(f_{n}\right)-\Phi_{\gamma, c_{2}}\left(\frac{n f_{n}(x)}{\left|\ell\left(f_{n}\right)\right|}\right) \leq C_{0}<\infty
$$

$$
n\left(\frac{\ell\left(f_{n}\right)}{\left|\ell\left(f_{n}\right)\right|}\right) \leq 1+C_{0}
$$

Notice that we can always assume $\ell\left(f_{n}\right)>0$, otherwise we replace $f_{n}$ by $-f_{n}$.

Part 2. This is an application of Theorem 1.1, p. 2 in [13], based on Proposition 3 and Lemma 1.

We have shown that the supremum is achieved for a function $h \in U_{\Phi_{\gamma, 1}}$. Since this function depends on $\mu$, we can write $h=h_{\mu}$ when needed. The first observation is that $0 \leq \ell(h)-\Phi_{\zeta, V}(h)<\infty$. The functional is nonnegative because it equals zero at $f=0$, and $h$ is a maximizer. On the other hand, $h \in U_{\Phi_{\gamma, 1}}$, and $\ell$ is a bounded functional, hence the linear part is finite. The functional $\Phi_{\zeta, V}(h)$ is nonnegative and as seen above, $0 \leq \Phi_{\zeta, V}(h) \leq \ell(h) \leq C\|h\|_{\Phi_{\gamma, 1}}$, which shows that $\Phi_{\zeta, V}(h)<\infty$.

Once we know that, we write $\ell(f)-\Phi_{\zeta, V}(f) \leq \ell(h)-\Phi_{\zeta, V}(h)$ for $f(s, x)=f_{\epsilon}(s, x)=$ $\left.h(s, x)+\epsilon \phi(s, x), \phi(s, x) \in C_{c}^{\infty}\left([0, t], \mathbf{T}^{d}\right)\right)$. Due to the boundedness of the smooth functions $\phi$, all $0 \leq \Phi_{\zeta, V}\left(f_{\epsilon}\right)<\infty$. The function $\zeta(x)$ is finite everywhere $(\operatorname{dom} \zeta=\mathbb{R})$, smooth, strictly convex, increasing and $\zeta(x)=0$ only if $x=0$. As a function of $\epsilon$, the functional $\ell\left(f_{\epsilon}\right)-\Phi_{\zeta, V}\left(f_{\epsilon}\right)$ from (4.5) has a maximum at $\epsilon=0$.

We want to prove that the functional is differentiable at $\epsilon=0$. Taylor's formula for $\zeta(a)=e^{a}-1-a, \zeta(a+\epsilon b)=\zeta(a)+\epsilon b \zeta^{\prime}(a)+\epsilon b \int_{0}^{1} \zeta^{\prime}(a+\lambda \epsilon b)-\zeta^{\prime}(a) d \lambda$ applied to $a=f(s, y)-f(s, x)$ and $b=\phi(s, y)-\phi(s, x)$ tells us that we have to calculate

$$
\lim _{\epsilon \rightarrow 0} \int_{0}^{t} \iint\left\{e^{f(s, y)-f(s, x)} \int_{0}^{1}\left[e^{\lambda \epsilon(\phi(s, y)-\phi(s, x))}-1\right] d \lambda\right\} V(x) \mu(s, d x) \mu(s, d y) d s=0 .
$$

For $M>1$ we have $e^{a} \leq e^{M}$ if $|a| \leq M$ and there exists a constant $c_{M}>0$ such that $e^{a} \leq c_{M} \zeta(a)$ for $|a|>M$, giving the bound $e^{a} \leq e^{2}+c_{2} \zeta(a)$ for $M=2$. This, and the fact that $\phi, \lambda$ are bounded, as well as $\Phi_{\zeta, V}(h)<\infty$ allow us to find an integrable upper 
bound independent of $\epsilon$ for the integrand in braces. Applying dominated convergence we are done.

By equation (4.4) with (4.9), we have shown that for a fixed $\mu(t, d x)$, the solution $h_{\mu}$ satisfies the weak equation (1.13), that is, for $\zeta^{\prime}(x)=e^{x}-1$

$$
\begin{array}{r}
\ell(\phi)=\int_{0}^{t} \int \nabla \phi(s, x) \cdot \nabla h(s, x) \mu(s, d x) d s \\
+\int_{0}^{t} \iint(\phi(s, y)-\phi(s, x)) \zeta^{\prime}(h(s, y)-h(s, x)) V(x) \mu(s, d x) \mu(s, d x) d s .
\end{array}
$$

Remark. Whenever $(h(s, y)-h(s, x)) \exp \{(h(s, y)-h(s, x))\}$ is integrable, reasoning along the lines of the maximization following equation (5.8), equation (4.9) allows us to write the rate function as $I_{d y n}(\mu)$ from (1.24).

\section{Lower Bound}

Denote by $P_{\rho, H}^{N}$ the law of the $N d$-dimensional process solving the martingale problem (1.3) - (1.5), where $\rho(x)$ is the density of the initial profile $\mu_{h}(d x)(1.9)$. In the present setting, $x_{i}(0), 1 \leq i \leq N$ are i.i.d. random variables with $x_{i}(0) \sim \rho(x) d x$. Accordingly, $P^{N}$ will denote the law of the process with $h \equiv 0$ and $\rho=\rho_{0}$. We can assume that $\rho=\rho_{0}$. Since the particles are i.i.d. at time $t=0$, in the general case we should add to (5.2) and (5.3) the relative entropy of the two initial profiles. is

The Radon-Nikodym derivative of $P_{\rho, H}^{N}$ with respect to $P^{N}$ over the time interval $[0, T]$

$$
\begin{gathered}
\frac{d P_{\rho, H}^{N}}{d P^{N}}=\exp \{H(T, \mathbf{x}(T))-H(0, \mathbf{x}(0)) \\
-\int_{0}^{T}\left(\partial_{s} H(s, \mathbf{x}(s))+\frac{1}{2} \sum_{i=1}^{N} \Delta_{x_{i}} H(s, \mathbf{x}(s))\right) d s \\
-\frac{1}{2} \int_{0}^{T} \sum_{i=1}^{N}\left|\nabla_{x_{i}} H(s, \mathbf{x}(s))\right|^{2} d s \\
\left.-\int_{0}^{T} e^{-H(s, \mathbf{x}(s))} \sum_{i=1}^{N}(N-1)^{-1} V\left(x_{i}(s)\right) \sum_{j \neq i}^{N}\left(e^{H\left(s, \mathbf{x}^{i j}(s)\right)}-e^{H(s, \mathbf{x}(s))}\right) d s\right\} .
\end{gathered}
$$

Hence the relative entropy normalized by a factor of $N^{-1}$ is

$$
\begin{gathered}
N^{-1} \mathcal{H}\left(P_{\rho, H}^{N}: P^{N}\right)=E_{P_{\rho, H}}\left[N^{-1} \log \left(\frac{d P_{\rho, H}^{N}}{d P^{N}}\right)\right]= \\
\left\langle h(T, \cdot), \mu^{N}(T, \cdot)\right\rangle-\left\langle h(0, \cdot), \mu^{N}(0, \cdot)\right\rangle-\int_{0}^{T}\left\langle\partial_{s} h(s, \cdot), \mu^{N}(s, d x)\right\rangle d s \\
-\frac{1}{2} \int_{0}^{T}\left(\left\langle\Delta h(s, \cdot), \mu^{N}(s, \cdot)\right\rangle+\left\langle|h(s, \cdot)|^{2}, \mu^{N}(s, \cdot)\right\rangle\right) d s \\
-\int_{0}^{T} \int_{\mathbf{T}^{d}} \int_{\mathbf{T}^{d}}\left(e^{h(s, y)-h(s, x)}-1\right) V(x) \mu^{N}(s, d y) \mu(s, d x) d s-\mathcal{E}_{1}(N, h),
\end{gathered}
$$


where $\mu^{N}(t, d x)$ is the empirical measure (1.8) and $\mathcal{E}_{1}(N, h)$ is an error term of order $N^{-1}$, depending on the supremum norm of the smooth function $h$.

Proposition 4. Let $\mu_{h}(\cdot, d x) \in \mathbf{D}\left([0, T], M_{1}\left(\mathbf{T}^{d}\right)\right)$ be the hydrodynamic limit of the empirical measures (1.8) corresponding to the drift $h \in C^{1,2}\left([0, \infty), \mathbf{T}^{d}\right)$, with initial profile $\mu_{h}(d x)=\rho(x) d x$, where $\rho(x)$ is bounded. Then

$$
\lim _{N \rightarrow \infty} N^{-1} \mathcal{H}\left(P_{\rho, H}^{N}: P^{N}\right)=I_{\text {dyn }}\left(\mu_{h}\right) .
$$

In addition, the explicit expression of $I_{d y n}(\mu)$ for $\mu=\mu_{h}$ is given by (5.5)-(5.7).

Remark. We note that $h \equiv 0$ implies that $I_{d y n}\left(\mu_{h}\right)=0$.

Proof. We know (Theorem 1) that under the law $P_{\rho, H}^{N}$, the process $\left\{\mu^{N}(\cdot, d x)\right\}_{0 \leq t \leq T}$ converges in probability, uniformly in time, to the solution to the PDE expressed in weak form in (1.13). Since the perturbation term $h(t, x) \in C^{1,2}\left([0, T], \mathbf{T}^{d}\right)$, the functional from the right hand side of $(5.2)$ over $\mathbf{D}\left([0, T], \mathbf{T}^{d}\right)$ is continuous and bounded. Combining the hydrodynamic limit equation in weak form for the particular choice of $\phi=h$ in (1.13) and (5.2), we obtain

$$
\begin{gathered}
\lim _{N \rightarrow \infty} N^{-1} \mathcal{H}\left(P_{\rho, H}^{N}: P^{N}\right)= \\
\frac{1}{2} \int_{0}^{T}\left\langle|\nabla h(s, \cdot)|^{2}, \mu_{h}(s, \cdot)\right\rangle d s \\
+\int_{0}^{T} \int_{\mathbf{T}^{d}} \int_{\mathbf{T}^{d}}(h(s, y)-h(s, x)) e^{h(s, y)-h(s, x)} V(x) \mu_{h}(s, d y) \mu_{h}(s, d x) d s \\
-\int_{0}^{T} \int_{\mathbf{T}^{d}} \int_{\mathbf{T}^{d}}\left(e^{h(s, y)-h(s, x)}-1\right) V(x) \mu_{h}(s, d y) \mu_{h}(s, d x) d s .
\end{gathered}
$$

We want to compare the limit (5.4) as a functional of the measure $\mu_{h}(\cdot, d x)$ to the value of $I_{d y n}\left(\mu_{h}\right)$, where $I_{d y n}(\mu)$ is the large deviations upper bound rate function defined in (1.24). With the notation established in (1.18), and (1.20)-(1.22),

$$
\begin{gathered}
\sup _{\phi \in C^{1,2}\left([0, T], \mathbf{T}^{d}\right)} J_{\phi}\left(\mu_{h}\right)=\sup _{\phi}\left\{\ell_{\phi}\left(\mu_{h}\right)-\frac{1}{2} \int_{0}^{T}\left\langle|\nabla \phi(s, \cdot)|^{2}, \mu_{h}(s, \cdot)\right\rangle d s\right. \\
\left.-\int_{0}^{T} \int_{\mathbf{T}^{d}} \int_{\mathbf{T}^{d}}\left(e^{\phi(s, y)-\phi(s, x)}-1\right) V(x) \mu_{h}(d y) \mu_{h}(d x) d s\right\} .
\end{gathered}
$$

Once again, reading the equation (1.13) satisfied by $\mu_{h}$, we can put the quantity to be maximized from (5.8) in the form

$$
\begin{gathered}
\frac{1}{2} \int_{0}^{T}\left[\left\langle|\nabla h(s, \cdot)|^{2}, \mu_{h}(s, \cdot)\right\rangle-\left\langle|\nabla h(s, \cdot)-\nabla \phi(s, \cdot)|^{2}, \mu_{h}(s, \cdot)\right\rangle\right] d s \\
+\int_{0}^{T} \int_{\mathbf{T}^{d}} \int_{\mathbf{T}^{d}}\left[(\phi(s, y)-\phi(s, x)) e^{h(s, y)-h(s, x)}\right. \\
\left.-\left(e^{\phi(s, y)-\phi(s, x)}-1\right)\right] V(x) \mu_{h}(s, d y) \mu_{h}(s, d x) d s .
\end{gathered}
$$


Denote $\alpha(p, q)=p e^{q}-\left(e^{p}-1\right)$ and notice that, as a function of $p \in \mathbb{R}, \alpha$ is a concave function with the only critical point $p=q$ where the maximum value $\sup _{p} \alpha(p, q)=\alpha(q, q)$. By substituting $p=\phi(s, y)-\phi(s, x)$ and $q=h(s, y)-h(s, x)$, we have proven (5.3).

Let $C^{\prime}\left([0, T], M_{1}\left(\mathbf{T}^{d}\right)\right)$ be the time-continuous probability - measure valued paths in $\left.C\left([0, T], M_{1}\left(\mathbf{T}^{d}\right)\right)\right)$ that are absolutely continuous with respect to the Lebesgue measure $d x$ on $\mathbf{T}^{d}$ ) and have Radon-Nikodym derivative $\left.v(s, x) \in C^{1,2}\left([0, T], \mathbf{T}^{d}\right)\right)$. To complete the proof of the lower bound, we have to prove the following statement.

Proposition 5. Let $\left.\mu(\cdot, \cdot) \in C\left([0, T], M_{1}\left(\mathbf{T}^{d}\right)\right)\right)$ be a time-continuous path of probability measures on $\left.\mathbf{T}^{d}\right)$ such that the functional $I_{d y n}(\mu)$ defined in the variational formula (4.5) is finite. Then (i) there exists a family of integrable functions $v(s, x)$ indexed by $s \in[0, T]$ for which $\mu(s, d x)=v(s, x) d x$, and (ii) there exists a sequence of measures $\mu_{n}(\cdot, d x) \in$ $\left.C^{\prime}\left([0, T], M_{1}\left(\mathbf{T}^{d}\right)\right)\right)$ converging weakly to $\mu(\cdot, d x)$ such that $\lim _{n \rightarrow \infty} I_{\text {dyn }}\left(\mu_{n}\right)=I_{d y n}(\mu)$.

Proof. Step 1. The rate function $I(\mu)$ from (1.23) is lower semi-continuous. The part due to the initial distribution $I_{\text {init }}(\mu)$ is the classical entropy, while $I_{d y n}(\mu)$ described in (5.8) and (4.5) is the supremum of continuous functionals (in the sense of the weak convergence of measures) indexed by smooth test functions $\phi$. The continuity of the linear part is evident, and the continuity of the nonlinear part is a consequence of the fact that $V$ is continuous and bounded, and that the map taking $\mu(\cdot, d x)$ into the direct product $\mu(\cdot, d x) \otimes \mu(\cdot, d y)$ is continuous. Due to the special form of $I_{d y n}(\mu)$, we may abuse notation and identify $\mu$ with $\mu \otimes \mu$, noticing that $I_{d y n}(\mu \otimes \mu)=I_{d y n}(\mu)$, in the sense that we shall consider all terms in (5.8) integrated against $\mu(s, d y)$ as well, without loss of generality.

First consider a convolution of $\mu \otimes \mu$ with $\rho_{\epsilon}(x) \otimes \rho_{\epsilon}(y)$, where $\rho_{\epsilon}$ is a smooth approximation of the identity with $\rho_{\epsilon}(x) \geq 0$, supp $\rho_{\epsilon} \subseteq[-\epsilon, \epsilon]^{d}$ and total mass equal to one. The convolution will be equal to

$$
\rho_{\epsilon}(x) \otimes \rho_{\epsilon}(y) * \mu(\cdot, d x) \otimes \mu(\cdot, d y)=\rho_{\epsilon}(x) * \mu(\cdot, d x) \otimes \rho_{\epsilon}(y) * \mu(\cdot, d y) .
$$

Denote $\mu_{\epsilon}(\cdot, d x)=\rho_{\epsilon}(x) * \mu(\cdot, d x)$, an absolutely continuous measure with density $v_{\epsilon}(\cdot, x)$. Since $\mu_{\epsilon} \Rightarrow \mu$ and $I(\cdot)$ is lower semi-continuous, we have $I(\mu) \leq \lim \inf I\left(\mu_{\epsilon}\right)$. The functional $I(\cdot)$ is translation invariant, due to the translation invariance of the underlying space $\left(\mathbb{R}^{d}\right.$ or the torus) and the fact that the set of test functions over which we maximize in (5.8) is invariant to a space shift. By convexity (supremum over linear functionals of $\mu \otimes \mu$ ) and translation invariance of the functional $I(\cdot), I\left(\mu_{\epsilon}\right)=I\left(\mu_{\epsilon} \otimes \mu_{\epsilon}\right) \leq I(\mu \otimes \mu)=I(\mu)$.

We can assume that $\mu(\cdot, d x)=v(\cdot, x) d x$ with $v$ a smooth function in $x$. For $\epsilon>0$, write $\mu_{\epsilon}=(1-\epsilon) \mu+\epsilon \mu^{1}$, where $\mu^{1}$ is a probability measure with a smooth density in both $t$ and $x$ bounded away from zero on a fixed time interval $[0, T]$, in other words, there exists $c=c(T)>0$ such that $v(t, x) \geq c(T)$ for all $0 \leq t \leq T$ and $x \in \mathbb{R}^{d}$. Once again $\mu_{\epsilon} \Rightarrow \mu$. Lower semi-continuity and the convexity of $I(\cdot)$ applied to the convex combination that defines $\mu_{\epsilon}$ prove that we can assume without loss of generality that $\mu$ has a smooth density in $x$ and is bounded away from zero on any finite time interval. A standard procedure (see section 10.6 of [7]) allows us to assume that the density $v$ is smooth in the time variable as well.

Step 2. We have shown in Theorem 4 that for any given $\mu(\cdot, \cdot)$ such that $I(\mu)<\infty$, there exists a function $h \in U_{\gamma, 1}$ such that $\mu$ satisfies equation (1.14) in weak form with $h$ 
appearing in the nonlinear part $\mathbf{a}_{h}(\mu)$ of the equation. Let $v$ be the density of the measure $\mu$. To conclude our argument for the lower bound we need to show that if $v \geq c_{0}>0, v$ smooth in $t$ and $x$ and $h$ satisfies in weak sense the equation

$$
-\nabla \cdot(v \nabla h)+\alpha(h)=g, \quad g:=-\partial_{t} v+\frac{1}{2} \Delta v, \quad \alpha(h):=\mathbf{a}_{h}(v) v
$$

with nonlinear part $\alpha(h)$ defined in (1.12), then $\left.h \in C^{1,2}\left([0, T], \mathbf{T}^{d}\right)\right)$. The explicit form of $\alpha(h)$ is

$$
\alpha(h)(t, x)=\int_{\left.\mathbf{T}^{d}\right)} \alpha_{0}(t, x, y) v(t, x) v(t, y) d x d y
$$

where

$$
\alpha_{0}(t, x, y)=e^{h(t, x)-h(t, y)} V(y)-e^{h(t, y)-h(t, x)} V(x) .
$$

From theorem 4, we have

$$
\int_{0}^{T} \int_{\left.\mathbf{T}^{d}\right)}|h(t, x)|^{2} d x d s<\infty, \quad \int_{0}^{T} \int_{\left.\mathbf{T}^{d}\right)}|\alpha(h)(t, x)| d x d s<\infty,
$$

which implies that $\left.h(t, \cdot) \in H^{1}\left(\mathbf{T}^{d}\right)\right)$, $t$-a.e.. We look at the Poisson equation $-\nabla \cdot(v \nabla h)=$ $g_{1}$, with $g_{1}=g-\alpha(h)$. Since $g$ is smooth, the right hand side $\left.g_{1}(t, \cdot) \in L^{1}\left(\mathbf{T}^{d}\right)\right), t$ almost everywhere. Note that we can use the Lebesgue reference measure due to the uniform lower bound away from zero of both $v$ and $V$. Other than in $d=1$, regularity results for the solution of such an equation are difficult. In $d=1$, either directly by integration or using Theorem 8.15 in [4], we obtain that

$$
\sup _{\left.x \in \mathbf{T}^{d}\right)}|h(t, x)| \leq c_{1}\left(\|h(t, \cdot)\|_{2}+\|\alpha(h)\|_{1}\right), \quad t-\text { a.e. . }
$$

In the following $c_{j}$ denote constants independent of the functions involved in the equation, and time and space variables.

In the variable $x$, the strongly elliptic equation $-\nabla \cdot(v \nabla h)=g_{1}$ with $\left.g_{1} \in L^{\infty}\left(\mathbf{T}^{d}\right)\right) \subseteq$ $\left.L^{2}\left(\mathbf{T}^{d}\right)\right)$ has $\left.H^{2}\left(\mathbf{T}^{d}\right)\right)$ solutions $(6.3[2])$. Since $g_{1}$ is a smooth function of $h$, we can bootstrap this argument and show that $h(t, \cdot) \in C^{\infty}\left(\mathbf{T}^{d}\right)$ ). Examining (5.9) and its detailed form (1.14) we see that $h$ is determined up to shifts by a function of $t$, that is we can replace $h$ by $h(t, x)-h(t, 0)$ without loss of generality. The upper bound in (5.13) is integrable in the variable $t$. The next goal is to show that it is actually uniformly bounded in $t$.

Notice that $h \alpha(h)$ is jointly integrable in $(t, x)$, due to the smoothness of $g$ and $|\nabla h(t, x)|^{2}$ being integrable in $(t, x)$. Multiply $(5.9)$ by $h$ and integrate in $x$. Let $\beta(p)=p e^{p}+e^{-1}$, with $\beta(p) \geq 0$.

Since $h$ is smooth in the $x$ variable, and the equation on $\mathbf{T}^{d}$ ) has periodic boundary conditions, after applying Schwarz's inequality on the right hand side,

$$
\int_{\left.\mathbf{T}^{d}\right)}|\nabla h(t, x)|^{2} d x+\int_{\left.\mathbf{T}^{d}\right)} \beta(h(t, y)-h(t, x)) d x d y \leq c_{2}\|h(t, \cdot)\|_{1}+c_{3} .
$$

Under periodic boundary conditions, $\|h\|_{2} \leq c_{4}\|\nabla h\|_{2}$, and $\|h\|_{1} \leq c_{5}\|h\|_{2}$. We obtain that $\|\nabla h\|_{2} \leq c_{6}$ and $\|\alpha(h)\|_{1} \leq c_{7}$, hence $\|h\|_{2} \leq c_{8}$. These inequalities refer to norms in the $x$ variable, but are uniform in $t$. This, together with (5.13) show that $h(t, x)$ has essential supremum independent of $t$ and $x$. 
The next step is to prove uniform bounds in both $t$ and $x$ for the time-variable discrete derivative $D^{\delta} h(t, x)=\delta^{-1}(h(t+\delta, x)-h(t, x)), \delta>0$ sufficiently small. The time interval $[0, T]$ is considered for arbitrary $T$, which implies that we can consider from the beginning a larger time interval $[0, T+1]$ and prove the regularity of $h$ on $[0, T]$. This takes enables us to make sense of $h(t+\delta, x)$ for any $t \in[0, T]$. Differentiation rules applying to the discrete derivatives are described in [3]. The argument is easier to follow if we formally differentiate (5.9) with respect to the time variable and work with $\partial_{t} h$ in the sense of distributions. However, at the moment, we do not know whether $\partial_{t} h$ is integrable, or, for that matter, if it is a function. To prove this, based on (5.10)-(5.11), denote

$$
\begin{array}{r}
D^{\delta} \alpha(h)(t, x)= \\
\int_{\left.\mathbf{T}^{d}\right)} D^{\delta}\left[\alpha_{0}(t, x, y)\right] v(t, x) v(t, y) d x d y+ \\
\left.\int_{\left.\mathbf{T}^{d}\right)} \alpha_{0}(t, x, y) D^{\delta}[v(t, x) v(t, y))\right] d x d y .
\end{array}
$$

The equation satisfied by $D^{\delta} h(t, x)$ is

$$
\begin{array}{r}
\nabla \cdot\left(v(t, x) \nabla\left(D^{\delta} h(t, x)\right)+\left(D^{\delta} v(t, x)\right) \nabla h(t, x)\right)= \\
\int_{\left.\mathbf{T}^{d}\right)} D^{\delta}\left[\alpha_{0}(t, x, y)\right] v(t, x) v(t, y) d y+ \\
\left.\int_{\left.\mathbf{T}^{d}\right)} \alpha_{0}(t, x, y) D^{\delta}[v(t, x) v(t, y))\right] d y-D^{\delta} g(t, x)+\mathcal{E}(\delta, t, x),
\end{array}
$$

where $|\mathcal{E}(\delta, t, x)| \leq c_{9}$ is the order of magnitude of the error term, withe $c_{9}$ independent of $(\delta, t, x)$ and $h$. Denoting $a \otimes b=a(x) g(y)$ for any functions $a(x), b(y)$, we multiply (5.18) by $D^{\delta} h(t, x)$ and integrate over $x$ to have

$$
\int_{\left.\mathbf{T}^{d}\right)} v\left|\nabla\left(D^{\delta} h\right)\right|^{2} d x+\int_{\left.\mathbf{T}^{d}\right)} \int_{\left.\mathbf{T}^{d}\right)}\left(D^{\delta} \alpha_{0}\right)\left(D^{\delta} h\right) v \otimes v d y d x=\int_{\left.\mathbf{T}^{d}\right)} \beta\left(D^{\delta} h\right) d x,
$$

with

$$
\beta=-\left(D^{\delta} v\right) \nabla h-\int_{\left.\mathbf{T}^{d}\right)} \alpha_{0}\left[\partial_{t} v \otimes v+v \otimes \partial_{t} v\right] d y+\left(D^{\delta} g\right)-\mathcal{E} .
$$

It is essential that the first bracket in the integrand of (5.19) is symmetric in $(x, y)$. We need to show that the second term of $(5.21)$ is nonnegative, that is

$$
\int_{\left.\mathbf{T}^{d}\right)} \int_{\left.\mathbf{T}^{d}\right)}(\alpha(t+\delta, x, y)-\alpha(t, x, y))(h(t+\delta, x)-h(t, x)) v(t, x) v(t, y) d y d x \geq 0 .
$$

We symmetrize the integrand

$$
\begin{gathered}
\frac{1}{2} \int_{\left.\mathbf{T}^{d}\right)} \int_{\left.\mathbf{T}^{d}\right)}(\alpha(t+\delta, x, y)-\alpha(t, x, y)) \times \\
((h(t+\delta, x)-h(t+\delta, y))-(h(t, x)-h(t, y))) v(t, x) v(t, y) d y d x
\end{gathered}
$$


and see that if we write $z_{1}=h(t+\delta, x)-h(t+\delta, y), z_{0}=h(t, x)-h(t, y)$, then the whole integral is

$$
\int_{\left.\mathbf{T}^{d}\right)} \int_{\left.\mathbf{T}^{d}\right)}\left[\left(e^{z_{1}}-e^{z_{0}}\right) V(y)-\left(e^{-z_{1}}-e^{-z_{0}}\right) V(x)\right]\left(z_{1}-z_{0}\right) v(t, x) v(t, y) d y d x \geq 0 .
$$

Noticing that $D^{\delta}$ and $\nabla$ commute, and that the last three functions appearing in (5.22) are uniformly bounded in $(t, x)$, while $\left|\left(D^{\delta} v\right) \nabla h\right|^{2}$ has an integral in $x$ independent of $t$. Applying a Sobolev inequality to (5.21), we bound below the norm of the gradient by the norm of the function. Schwarz inequality for (5.22) gives that, in the square norm with respect to $x$,

$$
\left\|D^{\delta} h(t, x)\right\|_{H^{1}} \leq c_{10},
$$

again with $c_{10}$ independent of $(t, x, \delta)$. As a consequence, letting $\delta \rightarrow 0$, we showed that $h(\cdot, x)$ is absolutely continuous, hence differentiable a.e., with derivative $\left.\partial_{t} h(t, \cdot) \in L^{2}\left(\mathbf{T}^{d}\right)\right)$. If

$$
\alpha_{1}(t, x, y)=e^{h(t, x)-h(t, y)} V(y)+e^{h(t, y)-h(t, x)} V(x),
$$

we define the bounded linear operators $\left.\left.\beta: L^{1,2}\left([0, T], \mathbf{T}^{d}\right)\right) \longrightarrow L^{1,2}\left([0, T], \mathbf{T}^{d}\right)\right)$

$$
\beta_{1}(u)(t, x)=\int_{\left.\mathbf{T}^{d}\right)} \alpha_{1}(t, x, y)(u(t, x)-u(t, y)) v(t, x) v(t, y) d y .
$$

At this point we can write the pde satisfied by $u(t, x)=\partial_{t} h(t, x)$ as

$$
\nabla \cdot\left(v \nabla u+f_{1}\right)=\beta_{1}(u)+g_{1}
$$

where

$f_{1}=\left(\partial_{t} v\right) \nabla h, \quad\left\|f_{1}\right\|_{2} \leq c_{11}, \quad g_{1}=-\partial_{t} g+\int_{\left.\mathbf{T}^{d}\right)} \alpha_{0}(\partial v \otimes v+v \otimes \partial v) d y, \quad\left\|g_{1}\right\|_{\infty} \leq c_{12}$.

We verify that $\beta(u)$ is positive definite in the sense that $\int \beta(u) u d x \geq 0$, which is easier to see directly but is also a consequence of (5.23). Since $\beta\left(\partial_{t} h\right)$ is square integrable in $x$ with uniform bound in $t$, we consider (5.24) as a simpler elliptic equation with right hand side equal to $g_{2}$ square integrable and uniformly bounded in $t$. Theorem 8.15 in [4] shows that $u=\partial_{t} h$ is uniformly bounded in $(t, x)$.

The next step of the proof shows that $u=\partial_{t} h$ is differentiable in $t$ and the derivative is bounded in both $t$ and $x$. The reasoning is essentially the same as for $u=\partial_{t} h$ itself. In order to avoid technical difficulties related to the fact that $\beta$ is a linear operator and does not fit exactly the classical theory - the corresponding case is an elliptic operator with a negative potential - we shall proceed as before, working with the discrete derivative $D^{\delta} u$ at first. However, it is useful to write down the formal equation satisfied by $\partial_{t} u(t, x)$.

Since equation (5.24) is satisfied weakly in $(t, x)$, we can write, in the sense of distributions

$$
\nabla \cdot\left(v \nabla\left(\partial_{t} u\right)+f_{2}\right)=\beta_{1}\left(\partial_{t} u\right)+g_{2},
$$

with

and, for

$$
f_{2}=\partial_{t} f_{1}+\left(\partial_{t} v\right) \nabla u, \quad\left\|f_{2}\right\|_{2} \leq c_{13}
$$

$$
g_{21}(t, x)=\int_{\left.\mathbf{T}^{d}\right)} \partial_{t} \alpha_{1}(t, x, y)(u(t, x)-u(t, y)) v(t, x) v(t, y) d y
$$




$$
g_{22}(t, x)=\int_{\left.\mathbf{T}^{d}\right)} \alpha_{1}(t, x, y)(u(t, x)-u(t, y)) \partial_{t}[v(t, x) v(t, y)] d y
$$

we have

$$
g_{2}(t, x)=\partial_{t} g_{1}(t, x)+g_{21}+g_{22}, \quad\left\|g_{2}\right\|_{2} \leq c_{14} .
$$

We note that the bounds in square norms are in fact true in the uniform norm.

Due to linearity, equation (5.25) will be satisfied by the discrete derivative $D^{\delta} u(t, x)$. All we have to verify is that $\int \beta\left(\partial_{t} u\right) \partial_{t} u d x \geq 0$. Following the same steps as before, we conclude that $\partial_{t} u=\partial_{t t} h$ is bounded uniformly in $(t, x)$. He have shown that $h(t, x), \partial_{t} h(t, x)$, as well as the derivatives in $x$ are continuous in $(t, x)$.

The last step is to show that the spatial derivatives of $\partial_{t} h$ are jointly continuous in $t$ and $x$. We know that the spatial derivatives for both $h$ and $\partial_{t} h$ exist as functions in $L^{2}\left(\mathbf{T}^{d}\right)$ ) with norms uniformly bounded in $t$. Both (5.9) and (5.24) are classical equations when regarded in $x$, since the integrals over $y$ yield uniformly bounded functions of time, independent of the space variable. The proof proceeds by differentiating in the space variable equation (5.24). The linear operator $\beta(u)$ reduces to a classical term $c(t, x) u$, with $c$ uniformly bounded in $(t, x)$. Reasoning as above, we obtain in fact that all space derivatives of $\partial_{t} h$ are continuous in $x$, with uniform bounds in $(t, x)$. To complete the continuity in $t$, we know already that $\partial_{t t} h$ is a bounded function for all $(t, x)$. In conclusion we note that by iterating the same procedure, it can be shown that $h$ is infinitely differentiable in $(t, x)$.

\section{REFERENCES}

[1] Burdzy, K., Hołyst, R., March, P. (2000)A Fleming-Viot particle representation of the Dirichlet Laplacian. Comm. Math. Phys. 214, no. 3.

[2] Evans, L.C. (1998) Partial Differential Equations. American Mathematical Society, Providence, R.I.

[3] Folland, G. B. (1976), Introduction to Partial Differential Equations, Mathematical Notes, Princeton University Press.

[4] Gilbarg, D., Trudinger, N. S. (1983), Elliptic Partial Differential Equations of Second Order. SpringerVerlag, Berlin, New York.

[5] Grigorescu, Ilie; Kang, Min (2004) Hydrodynamic limit for a Fleming-Viot type system. Stochastic Process. Appl. 110, no. 1, 111-143.

[6] Kipnis, C.; Landim, C. Scaling Limits of Interacting Particle Systems. Springer-Verlag, New York, 1999.

[7] Kipnis, C.; Landim, C. Scaling Limits of Interacting Particle Systems. Springer-Verlag, New York, 1999.

[8] Kipnis, C., Olla, S., Varadhan, S.R.S. (1989) Hydrodynamics and large deviations for simple exclusion processes. Comm. Pure and Appl. Math. XLII, 115-137.

[9] Jona-Lasinio, G.; Landim, C.; Vares, M. E. Large deviations for a reaction diffusion model. Probab. Theory Related Fields 97 (1993), no. 3, 339-361.

[10] Lax, P. Functional Analysis, John Wiley \& Sons, New York, 2002.

[11] Oelschläger, K. (1985) A law of large numbers for moderately interacting diffusion processes. Z. Wahrscheinlichkeitstheorie verw. Gebiete, vol 69, 279-322.

[12] Rao, M.M.; Ren, Z. D. Theory of Orlicz spaces, Marcel Dekker 1991.

[13] Struwe, M. Variational methods: applications to nonlinear partial differential equations and Hamiltonian systems. Third edition, Springer 2000.

Department of Mathematics, University of Miami, Coral Gables, FL 33124-4250

E-mail address: igrigore@math.miami.edu 